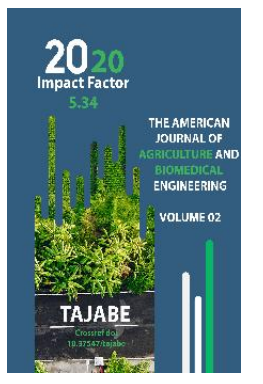

Journal Website: http://usajournalshub.c om/index,php/tajabe

Copyright: Original content from this work may be used under the terms of the creative commons attributes 4.0 licence.

\section{Analysis Of Scientific Research On Reproduction Of Species Of Trichograms In Biolaboratory}

\author{
R.Jumaev \\ Tashkent State Agrarian University And Andijan Branch Of Tashkent State Agrarian \\ University, Uzbekistan.
}

\author{
A.Rakhimova \\ Tashkent State Agrarian University And Andijan Branch Of Tashkent State Agrarian \\ University, Uzbekistan.
}

\title{
ABSTRACT
}

Based on the analysis of special literature, the article discusses specific techniques for improving the biological quality indicators of trichogramma when breeding it in biological laboratories. Special attention should be paid to the qualitative indicators of a trichogram when it is reproduced in a biological laboratory. These include: improving the technology of its breeding, storage and use; creation of optimal conditions for her upbringing in the process of reproduction in biolabs and biofactories; additional feeding of adults with various stimulants; constant introduction of mother cultures of Trichogramma and its laboratory host.

\section{KEYWORDS}

Trichogramma, biological method, reproduction of Trichogramma, scoop, grain moth, biological quality indicators of Trichogramma, hygrothermal breeding conditions, photoperiod, feeding of adults, renewal of mother material.

\section{INTRODUCTION}

In recent years, the biological method of combating harmful organisms has become generally accepted in the system of measures and is increasingly being used in agricultural production. A large amount of work has been done on the taxonomy, biology and ecology of entomophages (Alimukhamedov et al,
1986; Kimsanbaev et al., 1999; Khamraev, Nasretdinov, 2003). Representatives of entomophages and acariphages were registered in 16 orders, of which species of practical importance belong to 8 orders (Pomazkov and Zaets, 1997). 
Currently, the egg-eating parasite Trichogramma is the main agent and bioagent in the biological fight against the complex of harmful Lepidoptera on industrial and other crops $[1 ; 2 ; 5 ; 8]$.

The massive use of Trichogramma became possible with the introduction of industrial technology for its breeding into practice. However, the effectiveness of the practical use of trichogramma in natural conditions varies significantly from various factors, including weather. Thus, according to B.G. Degtyarev et al. (1988), along with an increase in the use of trichogramma, special attention of specialists should be paid to its quality indicators, on which the effectiveness of this work depends. These include: improving the technology of its breeding, storage and use; creation of optimal conditions for her upbringing in the process of reproduction in biolabs and biofactories; additional feeding of adults with various stimulants; constant introduction of mother cultures of trichogramma and its laboratory host; implementation of rational tactics and methods of introducing the egg eater into agrocenoses.

\section{MATERIALS AND METHODS}

The preservation of natural biological indicators of entomophages bred in laboratory conditions is of great importance in increasing the effectiveness of the biological method of protecting agricultural crops from pests. For these purposes, in laboratory conditions, technological conditions are observed (temperature, photoperiod, humidity, etc.). An important criterion for increasing the biological state of entomophages is the renewal of biomaterial from nature, their education on a natural host or victim. Violations of the technological process and year-round education of entomophages in the laboratory on a forced host leads to the loss of natural biological indicators $[4 ; 6 ; 7 ; 9 ; 12]$.

According to S.N. Alimukhamedov et al. (1986), the main biological indicators of the trichogramma quality include: fertility of females, the ratio of males and females in the offspring, survival (hatching from pupae,\%), the number of deformed or unsuitable individuals (\%), the lifespan of adults, infection of the eggs of the grain moth with trichogramma (\%), the number of oviparous females (\%).

A purposeful scientific search for methods to increase the viability of trichogramma, as well as obtaining standard products in the conditions of mass drug production of an entomophage in biofactories are impossible without the development of objective criteria for its quality. So, according to B.P. Adashkevich, in 1978 for the trichogramma reproduced in Uzbekistan, a standard was developed, where fertility should be - 30 eggs per female; oviparous females must be at least $90 \%$; the ratio of males and females is 1 : 1.5-1: 2; deformed individuals no more than 5\%; the survival rate is not less than $80 \%$, the life expectancy of adults at a temperature of 25 $290 C$ is 5-7 days; infestation of eggs of grain moths is at least $80 \%$ (Alimukhamedov et al., 1986). In 2007, the Scientific and Production Center of Agriculture of the Ministry of Agriculture and Water Resources of the Republic of Uzbekistan issued requirements for biological agents (entomophages) to combat pests of agricultural crops and methods for their control, where 5 main indicators and methods for their control are given for the trichogram.

Trichogramma refers to insects that easily change their quality indicators depending on the conditions of upbringing (Abashkin et al., 
1988). Failure to comply with the required breeding conditions - hygrothermal, mandatory diapause development in the autumn-winter period, feeding adults leads to a deterioration in the quality indicators of trichogramma, its vitality.

In laboratory conditions, the eggs of the grain moth are used as the host of the trichogramma. According to S.N. Alimukhamedov et al. (1986), with long-term education on them, the parasite loses some of its useful natural qualities. In particular, the overall body size, fertility, and life expectancy decrease, the number of deformed individuals in the offspring increases, and the sex ratio worsens. In this connection, below we will consider some methods of increasing the viability of Trichogramma.

Temperature is one of the most important factors controlling the activity of Trichogramma. Thus, the Chinese scientists, L. Liying, C. Yuehua, C. Zhonghua (1983) managed to show that the optimal conditions for the development of certain populations correlate with the climatic conditions of habitation. In their opinion, the effect of temperature on the growth and development of Trichogramma is specific for the species and population. With long-term cultivation in the laboratory, the response of the population to temperature effects is lost, and this obliges the constant renewal of the mother material due to collection in nature.

SN Alimukhamedov (1982), believes that in a hot and dry climate, populations of Trichogramma are formed with appropriate characteristics and the best results are obtained by using local species and populations that are ecologically adapted in the process of evolution to environmental conditions. For example, the Kashkadarya race formed under the conditions of Central Asia easily withstands a constant temperature of $35^{\circ} \mathrm{C}$ with a relative humidity of $30 \%$.

However, B.G. Degtyarev et al., (1988), believes that when breeding Trichogramma, an essential role is played by the education of the oviparasite in changeable hygrothermal conditions. Since the trichogramma, the breeding of which is carried out at constant temperatures, loses its daily rhythm, and when released to the field stations, it is active only under conditions similar to those under which it was diluted. So, when studying the possibility of breeding Trichogramma on other hosts, they developed a breeding technology for the cabbage scoop and obtained data on the effect of temperature on the performance of the technology. The data obtained indicate that the productivity of cabbage scoop cultivation at variable temperature is 1.5 times higher than at constant temperature $[7 ; 10 ; 11]$.

\section{RESULT AND DISCUSSION}

A similar opinion is shared by S.N. Alimukhamedov et al. (1986) that the dilution of Trichogramma at constant temperatures and air humidity leads to the "pampering" of the Trichogramma when the parasite is released into the fields, as a result they turn out to be weakly active, they do not tolerate changes in day and night temperatures, which affects their effectiveness. In trichogram shops, the temperature during the day must be kept within 25-300, at night - about $160 \mathrm{C}$.

An important factor that determines the duration of the development of trichogramma is the photoperiod. At different photoperiods, the duration of development from egg to adult emergence is not the same. According to the data cited by V.A. Zaslavsky (1984), during extreme short-day and long-day photoperiods, not only the time required for completion of insect development increases, 
but also the synchronization of emergence decreases. So, with a photoperiod of 16-20 hours, the beginning of the emergence of the adult Trichogramma is noted after 14-16 hours, and the peak of the flight is observed after 1517 hours. In addition, the photoperiod affects the initial fertility. Thus, in the species T.evanescens, during development on a grain moth, the number of eggs in the oviducts of females that developed at a temperature of $20^{\circ} \mathrm{C}$ and a short day (12 hours) averaged 17.524.7 , and in a long day (20 hours) - $24,7-28.6$ eggs (Zaslavsky et al., 1988).

Another effective method for increasing the viability of Trichogramma is feeding adults with a $20 \%$ solution of sucrose or honey (Alimukhamedov et al., 1986, Degtyarev et al., 1988). It is proved that feeding adult females of Trichogramma 20\% sugar syrup increases their fertility by 1.5-2 times, and life expectancy up to 10-15 days. According to N.I. Ignatko (1983), one-time feeding with a $20 \%$ sugar solution in combination with the education of trichogramma in natural conditions increases its fertility by $33.9-40.2 \%$. The value of feeding also affects the transfer of a trichogram from a natural host to a laboratory one. As noted by Sh.M. Greenberg and A.F. Rusnak (1985), the proportion of females that left offspring in the eggs of a grain moth at the first contact usually does not exceed $30-40 \%$. The negative effect of the transfer can be removed due to carbohydrate dressings. Since, during the first days of life, females really do not perceive the grain moth as a host. Without feeding, they die in a few days without leaving offspring. The females begin to infect the eggs of the grain moth only a few days later. This reserve of time is created by feeding.

One of the most important methods of increasing the efficiency of using laboratory populations of Trichogramma is the renewal of mother material. As pointed out by S.N. Alimukhamedov et al. (1986), when trichogramma reproduces on sitotroga eggs, the parasite from generation to generation loses its natural biological characteristics, which leads to a sharp decrease in its reproductive abilities. So, the fertility of trichogramma after 3 generations decreases by $50-60 \%$, and after 5 generations - by $70-80 \%$ and reaches $10-15$ eggs per female, which is half the established standard $[3 ; 5 ; 9]$.

\section{CONCLUSION}

The classical method recommended by S.N. Alimukhamedov et al. (1986), is that scoop butterflies are caught with a light trap throughout the growing season. A plastic bag filled with paper shavings is suspended at the bottom of the light trap cone, which allows the butterflies to stay alive until the morning. Every day, scoop butterflies are packaged in 15 copies. regardless of the floor in liter glass jars with accordions made of white filter paper. The jars are covered with gauze from above. The butterflies are fed with $20 \%$ sugar syrup and placed in the dark at a temperature of 25300C. Every morning, filter paper and lids are changed, dead butterflies are removed, replacing them with new ones. Usually, 300500 scoop eggs are obtained from one can per day. The accordions are hung on alfalfa, tomatoes, cabbage, corn, placing them in the shade of plants. After 3-4 days, they are removed, transferred to the laboratory and placed in one liter glass jars, several pieces at a temperature of $27-300 \mathrm{C}$ and a humidity of at least 70\%. After another 2-3 days, caterpillars of the scoop will begin to hatch from the uninfected eggs, and the infected ones will begin to darken. The latter are cut out and 2030 pieces are placed in large biological test tubes, covered with mill gas and placed in 
desiccators until the trichogramma leaves. It can be noted that $250-400$ specimens are obtained from each trap in 10 days. natural trichogramma of high quality. In the future, the trichogramma is propagated according to the generally accepted method. In JulyAugust, when populations of natural trichogramma accumulate, parasites infect up to $5 \%$ or more of the hanging eggs of scoops, which is enough to change the mother culture. indicators of trichogramma when breeding an egg eater in biological laboratories.

\section{REFERENCES}

1. Abashkin A.S., Grinberg Sh.M., Gorban V.P. State of the issue and prospects for the production of trichogramma in biofactories. // Trichogramma in plant protection. -M., 1988 - p. 3-13.

2. Alimukhamedov S.N. The current state of the art of integrated protection of cotton from pests, diseases and weeds. // Abstracts of the report. to the All-Union. Seminar-meeting on the experience of introducing a biological method into an integrated system for protecting cotton from pests and diseases. - Tashkent, 1982 - p. 7-11.

3. Alimukhamedov SN, Adashkevich B., Adylov Z, Khodzhaev Sh. // Biological method of combating the main pests of cotton - Tashkent, Mekhnat, 1986 - p.3065.

4. Grinberg Sh.M., Rusnak A.F. Modern trends in mass breeding of trichogramma /l Application of trichogramma and biological products in integrated pest control - Sofia, 1985 - pp. 36-44.

5. Degtyarev BG, Tsybulskaya GN, Yanishevskaya LV, et al. Problems of mass breeding of Trichogramma and its owners
// Trichogramma in plant protection. $-\mathrm{M}$., 1988 - p.13-22.

6. Zaslavsky V.A. Photoperiodic and temperature control of insect development. - L., Science, 1984 - 180 p.

7. Zaslavsky V.A., Mai Fu Kwi, Umarova T.Ya. Physiological reactions controlling the development and reproduction of trichogramma. // Trichogramma in plant protection. -M., 1988 - pp. 35-45.

8. Ignatko N.I. Techniques for improving the storage and breeding technology of trichogramma, increasing the efficiency of its use in the fight against pests of agricultural crops. Author's abstract. dis. Candidate of Agricultural Sciences - Kiev, $1983-24 \mathrm{p}$.

9. Kimsanboev X.X, NurmuhamedovD. N. OlmasboevaR. Sh, Rashidov M.l., Sulaymonov B.A., Yusupov A. X. Fertilization, storage and application of trichogram. -Tashkent, Teacher, 1999 - 34 p.

10. Liying L., Yuehua Ch., Ronghua Ch. The effect of temperature on the growth and development of the red-eyed wasp Trichogrammaspp. At the intraspecific and interspecific levels // Natural enemies of insects. 1983. No.1 - p.1-5

11. Requirements for biological agents (entomophages) for pest control of agricultural crops and methods for their control. - Tashkent, 2007-19 p.

12. Khamraev A.Sh., Nasriddinov K. Biological protection of plants.- Tashkent, People's heritage, 2003.-287 p. (Uzb.). 